\title{
Quantum Thermal Conductance of Electrons in a One-Dimensional Wire
}

\author{
O. Chiatti, ${ }^{1}$ J. T. Nicholls, ${ }^{1}$ Y. Y. Proskuryakov, ${ }^{1}$ N. Lumpkin, ${ }^{2}$ I. Farrer, ${ }^{2}$ and D. A. Ritchie ${ }^{2}$ \\ ${ }^{1}$ Department of Physics, Royal Holloway, University of London, Egham, Surrey TW20 OEX, United Kingdom \\ ${ }^{2}$ Department of Physics, Cavendish Laboratory, J J Thomson Avenue, Cambridge CB3 OHE, United Kindgom
}

(Received 24 April 2006; published 1 August 2006)

\begin{abstract}
We use an electron thermometer to measure the temperature rise of approximately $2 \times 10^{5}$ electrons in a two-dimensional box, due to heat flow into the box through a ballistic one-dimensional (1D) constriction. Using a simple model we deduce the thermal conductance $\kappa\left(V_{g}\right)$ of the 1D constriction, which we compare to its electrical conductance characteristics; for the first four 1D subbands the heat carried by the electrons passing through the wire is proportional to its electrical conductance $G\left(V_{g}\right)$. In the vicinity of the 0.7 structure this proportionality breaks down, and a plateau at the quantum of thermal conductance $\pi^{2} k_{B}^{2} T / 3 h$ is observed.
\end{abstract}

PACS numbers: 72.20.Pa, 73.23.Ad, 73.50.Lw, 73.63.Nm

The fabrication of tunable constrictions in high mobility two-dimensional electron gases (2DEGs) has allowed the study of transport in clean one-dimensional (1D) wires. This leads to an important result [1,2] in mesoscopic physics: the observation of quantized plateaus in units of $G_{0}=2 e^{2} / h$ in the electrical conductance characteristics $G\left(V_{g}\right)$. In the only reported measurements [3] of the thermal conductance in similar GaAs based wires, plateaulike structures in $\kappa\left(V_{g}\right)$ were obtained as the 1D subbands were depopulated. In this Letter we refine the device and technique used previously [3] so that we can directly compare $\kappa\left(V_{g}\right)$ to electrical conductance measurements $G\left(V_{g}\right)$. Such a comparison is important because if both charge and energy are transported by electrons, there is a universal relation between $\kappa$ and $G$ known as the Wiedemann-Franz (WF) relation

$$
\frac{\kappa}{G T}=\frac{\pi^{2} k_{B}^{2}}{3 e^{2}}=L_{0}
$$

where $L_{0}$ is the Lorenz number and $T$ is the temperature. Equation (1) predicts that the observation of conductance plateaus (in units of $G_{0}$ ) should be matched by steps in the thermal conductance, quantized in units of $L_{0} T \times G_{0}=$ $\left(1.89 \times 10^{-12} \mathrm{~W} / \mathrm{K}^{2}\right) T$. In addition it has been theoretically shown that in $1 \mathrm{D}$ the quantization of the thermal conductance is universal for thermal carriers that obey boson [4], fermion, or anyon statistics [5]. Careful low temperature measurements on suspended dielectric wires have demonstrated [6] this universality for phonons (bosons).

We find that over most of the gate voltage characteristics of a 1D wire the thermal conductance $\kappa$ follows the electrical conductance $G$ according to Eq. (1). In the vicinity of the 0.7 structure there is a breakdown of the WF relation, giving an unexpected plateau in thermal conductance at $L_{0} T\left(G_{0} / 2\right)$. The 0.7 structure is a reproducible feature observed near $0.7 G_{0}$ in the conductance characteristics $G\left(V_{g}\right)$ of clean 1D samples. There is growing experimental evidence that it has a spin origin: there is an enhanced $g$ factor as the 1D subbands are depopulated [7], in a strong parallel magnetic field the 0.7 structure evolves [7-9] into the usual spin-split plateau at $G_{0} / 2$, and the 0.7 structure moves down to $G_{0} / 2$ as the density is decreased [10,11]. Recent shot-noise measurements [12] show that the 0.7 structure could be interpreted assuming two conducting channels which have different transmission probabilities. Unusually the 0.7 structure becomes stronger with temperature [7], a result most clearly demonstrated [13] in etched samples which have strong lateral confinement. The 0.7 structure could be interpreted $[7,8]$ as a spontaneous static spin polarization within the lowest $1 \mathrm{D}$ subband, but the plateaulike structure occurs at $0.7 G_{0}$ rather than at $e^{2} / h$, and moreover the temperature dependence suggests that the 0.7 structure is not a ground-state property. There is no single mechanism for the 0.7 structure, static [7], dynamic [9], or otherwise, that can account for all the experimental observations.

In previous thermal conductance [3] and thermopower $[14,15]$ measurements, electrons on one side of the 1D constriction were heated above the lattice temperature $T_{L}$ by passing a current $I_{H}$ through the electron gas in that region. The electron-electron scattering rate is much faster than all other rates, and so electrons in the heating channel equilibrate at a local temperature $T_{H}=T_{L}+\Delta T$. We modify the device of Molenkamp et al. [3] by introducing a closed electron box, whose temperature $T_{\mathrm{box}}$ is measured from the thermopower in the linear regime $[14,16]$. The electrons in the closed box have a well-defined temperature and for a given $I_{H}$ it produces larger thermovoltages than the more open structures, for example, when constriction $C$ is not defined.

We present results for a sample fabricated from a wafer grown by molecular beam epitaxy. The 2DEG is $100 \mathrm{~nm}$ below the sample surface, with a carrier density of $3 \times$ $10^{11} \mathrm{~cm}^{-2}$ and a mobility of $5 \times 10^{6} \mathrm{~cm}^{2} / \mathrm{Vs}$. To avoid switching effects, the sample was cooled down with a positive voltage applied to the gates [17]. Three other 
samples showed results similar to those presented here. Figure 1 shows a schematic of the $6 \mu \mathrm{m} \times 10 \mu \mathrm{m}$ box, about which there are three split-gates, each with a gap that is $0.5 \mu \mathrm{m}$ long and $0.65 \mu \mathrm{m}$ wide. A current $I_{H}$ of frequency $f=32 \mathrm{~Hz}$ heats electrons in the heating channel to a temperature $T_{H}=T_{L}+\Delta T$. Hot electrons can enter the box through $A$, the sample constriction, and leave via constrictions $B$ and $C$. In steady state the heat flows in and out of the box are equal:

$$
\kappa_{A}\left(T_{H}-T_{\text {box }}\right)=\left(\kappa_{B}+\kappa_{C}\right)\left(T_{\text {box }}-T_{L}\right),
$$

and the increase in the box temperature is given by

$$
\delta T=\frac{\kappa_{A}}{\kappa_{A}+\kappa_{B}+\kappa_{C}} \Delta T,
$$

where $\kappa_{i}$ is the thermal conductance of the $i$ th constriction. $B$ and $C$ are known as the thermometer and reference constrictions, and are used to measure the temperature $T_{\text {box }}$. The temperature rise $\delta T$ within the box generates a thermovoltage in both $B$ and $C$; however, if constriction $C$ is put on a plateau its contribution is zero [14]; the thermovoltage measured at frequency $2 f$ is solely due to constriction $B$

$$
V_{\mathrm{th}}^{\text {box }}=S_{B}\left(T_{\text {box }}-T_{L}\right)=S_{B} \delta T .
$$

The thermopower $S_{B}$ is proportional to the average of the temperatures on either side of constriction $B$, allowing

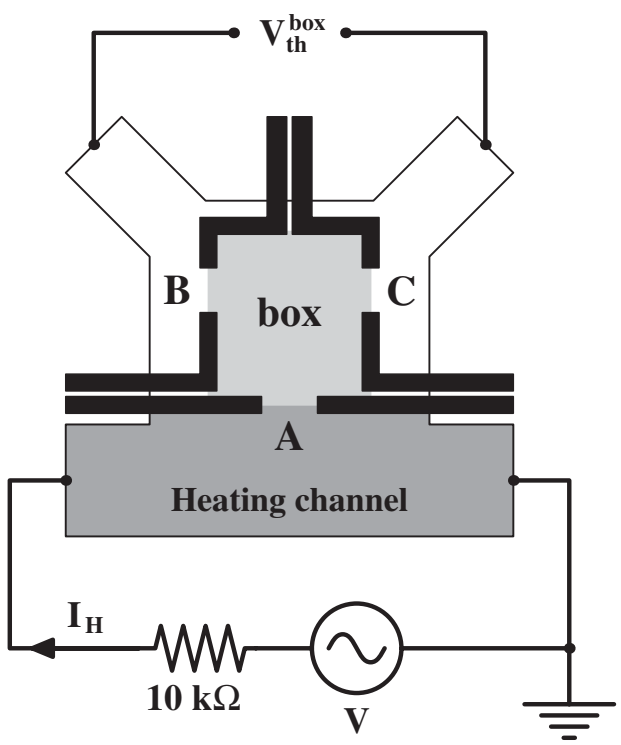

FIG. 1. Schematic of the device and setup for thermal conductance measurements. Electrons in the heating channel are heated to $T_{H}=T_{L}+\Delta T$ by a current $I_{H}$; hot electrons pass through the sample constriction $(A)$ into the box, where the temperature $T_{\text {box }}=T_{L}+\delta T$ is determined by the thermovoltage $V_{\text {th }}^{\text {box }}$ generated across the thermometer and reference constrictions ( $B$ and $C$, respectively). Electrons can only pass through the constrictions labeled $A, B$, and $C$; the much narrower channels are pinched off.
Eq. (4) to be rewritten as

$$
V_{\mathrm{th}}^{\mathrm{box}}=c_{B}\left(T_{\text {box }}^{2}-T_{L}^{2}\right),
$$

where the calibration constant, $c_{B} \approx 15 \mu \mathrm{V} / \mathrm{K}^{2}$, is determined by dc source-drain voltage measurements of the subband spacing [14]. When constriction $A$ is not defined, the electron thermometer is in direct contact with the heating channel; the magnitude of thermovoltage at this gate voltage, $V_{\text {th }}^{H}$, is a measure of $T_{H}$ through the relation

$$
V_{\mathrm{th}}^{H}=c_{B}\left(T_{H}^{2}-T_{L}^{2}\right) .
$$

Equations (3) and (4) show that measurements of $V_{\mathrm{th}}^{\mathrm{box}}\left(V_{g}\right)$, and hence $\delta T$, lead directly to the thermal conductance $\kappa_{A}\left(V_{g}\right)$, provided we know $\Delta T$ and we make certain assumptions about $\kappa_{B}$ and $\kappa_{C}$.

Figure 2 shows raw thermovoltage $V_{\mathrm{th}}^{\text {box }}\left(V_{g}\right)$ data taken as the gate voltage $V_{g}$ of constriction $A$ is swept, while constrictions $B$ and $C$ are set at $G_{B}=1.5 G_{0}$ and $G_{C}=G_{0}$, respectively. The steps in $V_{\text {th }}^{\text {box }}$ are aligned with those in the conductance $G_{A}$ (top trace), confirming that the structure in $V_{\text {th }}^{\text {box }}$ is due to the $1 \mathrm{D}$ subbands. Traces (a)-(d) were obtained with heating currents $I_{H}=0.2-1 \mu \mathrm{A}$, and the corresponding temperature rise in the heating channel $\Delta T=15-100 \mathrm{mK}$ was calculated using Eq. (5).

The thermovoltage $V_{\mathrm{th}}^{\text {box }}$ shown in Fig. 2 follows the shape of the conductance characteristics $G_{A}\left(V_{g}\right)$; as well as the steps, the definition of constriction $A$ at $V_{g} \approx 0.3 \mathrm{~V}$ can be seen in all the traces [18]. At $V_{g}=0.5 \mathrm{~V}$ both constriction $A$ and the box are not defined, and the electron thermometer is in direct contact with the heating channel;

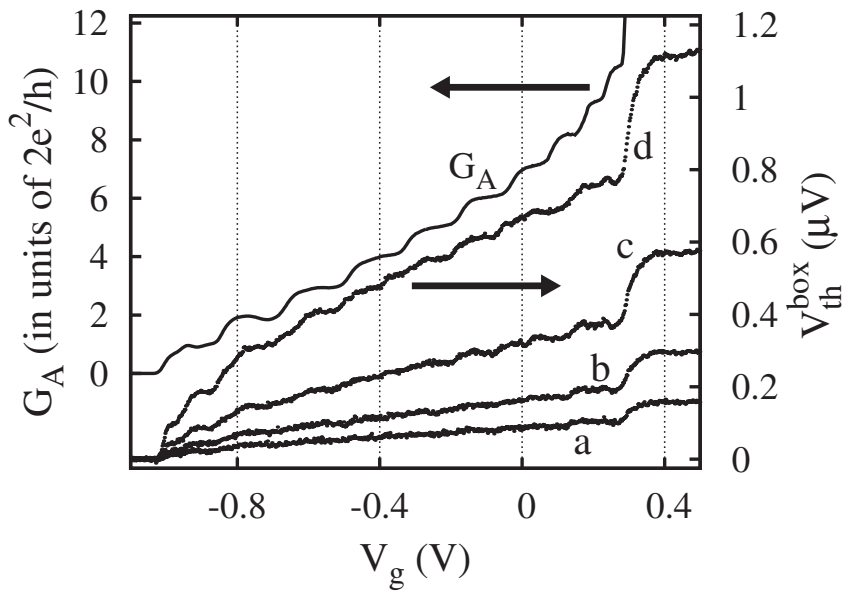

FIG. 2. The thermovoltage $V_{\mathrm{th}}^{\mathrm{box}}$ of the electron box together with the conductance $G_{A}$, measured at a lattice temperature $T_{L}=0.27 \mathrm{~K}$ using heating currents of (a) $I_{H}=0.2 \mu \mathrm{A}$, (b) $0.3 \mu \mathrm{A}$, (c) $0.5 \mu \mathrm{A}$, and (d) $1 \mu \mathrm{A}$. The corresponding temperature rise $\Delta T=15,30,55$, and $100 \mathrm{mK}$, respectively, were estimated using a source-drain calibration of the subbands. At $T_{L}=0.27 \mathrm{~K}$ the thermovoltage approximately scales with $I_{H}^{2}$ up to $I_{H}=1 \mu \mathrm{A}$. 
we define $V_{\mathrm{th}}^{\mathrm{box}}\left(V_{g}=0.5 \mathrm{~V}\right)=V_{\mathrm{th}}^{H}$, and $T_{H}$ is obtained using Eq. (6). Energy loss from the heated electrons to the lattice via the electron-phonon interaction $\left(\propto T^{5}\right)$ have been quantified [14], and can be neglected for $T<0.5 \mathrm{~K}$. To ensure that the measurements of both $\kappa_{A}$ and $V_{\text {th }}^{\text {box }}$ are in the linear regime, the heating in the channel is limited to $\Delta T<100 \mathrm{mK}$.

It is not possible to measure $\kappa_{A}, \kappa_{B}$, and $\kappa_{C}$ independently, and so we assume that the thermal conductance of each constriction $\kappa_{j}(j=A, B$ or $C)$ is proportional to its electrical conductance $G_{j}$,

$$
\kappa_{j}=\alpha_{j} G_{j} \bar{T},
$$

where $\alpha_{j}$ is a constant and $\bar{T}$ is the average of the temperatures on either side of the $j$ th constriction. If the WF ratio is the same for all three constrictions, $\alpha_{A}=\alpha_{B}=$ $\alpha_{C}=\alpha$, Eq. (2) can be written as

$$
\tilde{G}_{A}=\left(G_{B}+G_{C}\right) \frac{T_{\text {box }}^{2}-T_{L}^{2}}{T_{H}^{2}-T_{\mathrm{box}}^{2}}=\left(G_{B}+G_{C}\right) \frac{V_{\mathrm{th}}^{\mathrm{box}}}{V_{\mathrm{th}}^{H}-V_{\mathrm{th}}^{\mathrm{box}}},
$$

where $V_{\mathrm{th}}^{\mathrm{box}}\left(V_{g}\right)$ is measured as a function of the gate voltage on $A$, and $V_{\mathrm{th}}^{H}$ is the thermovoltage when constriction $A$ is not defined. By fixing $G_{B}$ and $G_{C}$ at known conductances (usually constriction $B$ is on a riser and $C$ is on a plateau) the quantities $V_{\text {th }}^{H}$ and $V_{\mathrm{th}}^{\text {box }}$ on the right side of Eq. (8) can be measured. The left side of Eq. (8) is written as $\tilde{G}_{A}$, which is the expected conductance of constriction $A$ as derived from thermal measurements. $\tilde{G}_{A}\left(V_{g}\right)$ has the dimensions of conductance, and will be compared to the electrical conductance $G_{A}\left(V_{g}\right)$.

Because of their different grounding requirements $G_{A}$ and $\tilde{G}_{A}$ are measured on different gate sweeps, nevertheless their pinch-off voltages agree to within $20-30 \mathrm{mV}$. Figure 3(a) shows traces of $\tilde{G}_{A}$ (offset horizontally) compared to the electrical conductance $G_{A}$, where the thermal data is taken with constriction $B$ fixed at $G_{B}=1.5 G_{0}$ and the reference constriction $C$ is set at $G_{C}=5 G_{0}$ [trace (i)], $4 G_{0}$ [trace (ii)], and $3 G_{0}$ [trace (iii)]. The increase in the box temperature, $\delta T$, as determined by Eq. (3) is different for each of the three traces, yet the same thermal conductance behavior for constriction $A$ is obtained, showing the validity of the technique. In comparison to $G_{A}$, which shows conductance plateaus at multiples of $G_{0}=2 e^{2} / h$, there are three plateaus in $\tilde{G}_{A}$ which occur (to within $10 \%-$ $15 \%)$ at similar multiples of $G_{0}$. The conductance characteristics $G_{A}\left(V_{g}\right)$ in Fig. 3(a) show a 0.7 structure that increases in strength with temperature; there is, however, no corresponding 0.7 structure in the $\tilde{G}_{A}\left(V_{g}\right)$ traces.

Figure 3(b) shows in detail the conductances closer to pinch off, where again for clarity the $\tilde{G}_{A}$ traces have been shifted to the right. The thermal conductances were measured at the lattice temperature $T_{L}=0.27 \mathrm{~K}$ for different

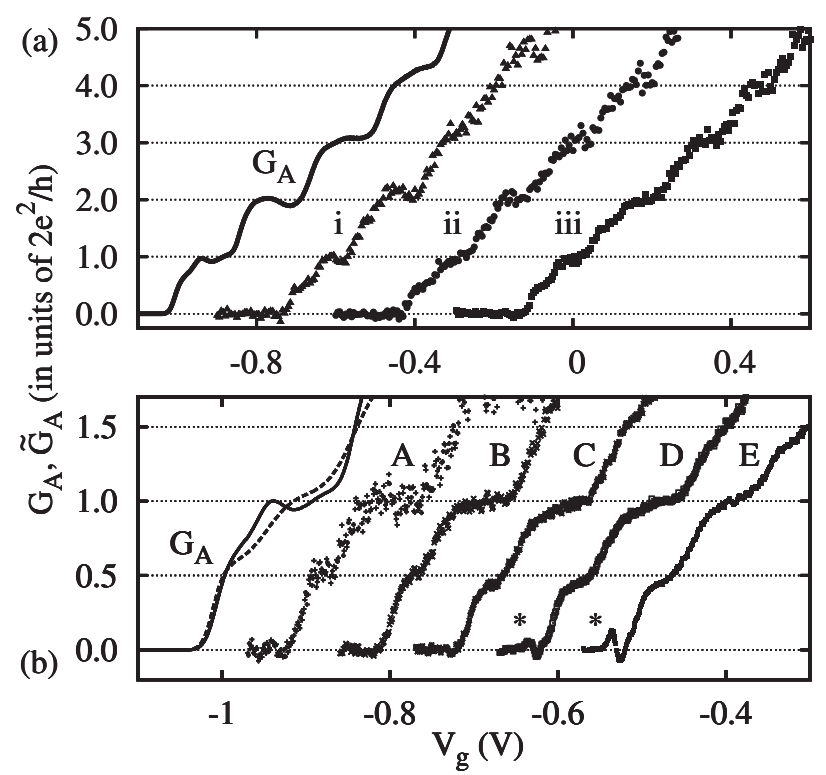

FIG. 3. (a) The thermally derived conductance $\tilde{G}_{A}\left(V_{g}\right)$ and electrical conductance $G_{A}\left(V_{g}\right)$ of constriction $A$ at $T_{L}=$ $0.27 \mathrm{~K}$. Left solid line: conductance $G_{A}\left(V_{g}\right)$. Measurements of $\tilde{G}_{A}\left(V_{g}\right)$ were obtained with $I_{H}=1 \mu \mathrm{A}, G_{B}=1.5 G_{0}$, and $G_{C}=$ $5 G_{0}$ [trace (i)], $G_{C}=4 G_{0}$ [trace (ii)], and $G_{C}=3 G_{0}$ [trace (iii)]. For clarity successive traces have been shifted by $0.3 \mathrm{~V}$ to the right. (b) Close-up of the thermal and electrical conductances near pinch off, showing that at the gate voltage corresponding to the 0.7 structure, $\tilde{G}_{A}$ exhibits a half plateau. Far left traces: $G_{A}\left(V_{g}\right)$ at $T_{L}=0.3 \mathrm{~K}$ (solid line) and $T_{L}=1.2 \mathrm{~K}$ (dashed line). Traces $A-E: \tilde{G}_{A}\left(V_{g}\right)$ at $T_{L}=0.27 \mathrm{~K}$ for heating currents $I_{H}=0.2,0.4,1,2$, and $3 \mu \mathrm{A}$; the corresponding $\Delta T$ are calculated to be $26,66,133,193$, and $234 \mathrm{mK}$. The $\tilde{G}_{A}\left(V_{g}\right)$ traces $A$ and $B$ were obtained with $G_{B}=1.5 G_{0}$ and $G_{C}=3 G_{0}$; traces $C-E$ were obtained with $G_{B}=1.5 G_{0}$ and $G_{C}=2 G_{0}$. The small spikes in traces $D$ and $E$ close to pinch off (marked with a *) only occur at high $I_{H}$, and are due to a capacitative coupling that can be avoided if dc measurements are performed.

heating currents. At the lowest $I_{H}(\operatorname{trace} A)$ there is a discernible half plateau at $e^{2} / h$ in $\tilde{G}_{A}$, which corresponds in gate voltage to the 0.7 structure in $G_{A}$. The noise on the half-plateau in trace $A$ is due to fluctuations in the measured voltage, $V_{\mathrm{th}}^{\mathrm{box}}=0.019 \pm 0.002 \mu \mathrm{V}$, and the associated value of the thermal conductance is $\tilde{G}_{A}=(0.54 \pm$ $0.06) G_{0}$. Therefore, the plateaus in $\tilde{G}_{A}$ can be measured with an accuracy better than 10\%; the corresponding conductance plateaus in $G_{A}$ are determined with greater accuracy $(<5 \%)$. With increasing $I_{H}$, traces $B$ to $E$ show less noise in $\tilde{G}_{A}$, and the half plateau in the thermal conductance is better resolved and its quantized value drifts down to $0.45 G_{0}$, a result that was obtained in all four samples and is reminiscent of the downward trend of the height of the 0.7 structure with temperature $[7,13]$. This unexpected half plateau in the thermal conductance was measured both in the linear regime, $\left(T_{H}-T_{\text {box }}\right) / T_{L} \sim 0.1$, but also persists into the nonlinear regime where $\left(T_{H}-T_{\text {box }}\right) / T_{L} \sim 0.7$. 
When the half plateau in the thermal conductance is just distinguishable, the heat flow through constriction $A$ is $\dot{Q}=$ $\kappa\left(T_{H}-T_{\text {box }}\right) \approx 6 \mathrm{fW}$, more than 2 orders of magnitude smaller than previous measurements [3].

In the single-particle picture a breakdown of the WF relation is possible if the transmission probability $t(E)$ of the constriction varies rapidly compared to the thermal energy difference across the constriction; however, calculations show [19] that for the realistic saddle-point potential model of the constriction there is no breakdown. First we discuss our thermal results for high conductances, $G>$ $G_{0}$, where single-particle theory prevails. Figure 3(a) shows the measured behavior with no adjustable parameters - there is an alignment of the first three conductance plateaus $\left(G_{A}=G_{0}, 2 G_{0}\right.$, and $\left.3 G_{0}\right)$ with those in $\tilde{G}_{A}$, where the latter quantity is calculated from Eq. (8) assuming that all three constrictions behave similarly. The observed alignment cannot be interpreted as an absolute confirmation of Eq. (1) because it is not known whether the constant of proportionality $\alpha$ [see Eq. (7)] is equal to the Lorenz number $L_{0}$. However, in the light of theoretical predictions [5] for the quantization of $\kappa$ for electrons, there is no reason why the WF relation should not be obeyed on the plateaus $G>G_{0}$.

Luttinger liquid behavior is expected in long clean 1D wires, and the resulting spin-charge separation causes the electrical and thermal conductances to behave differently, giving a breakdown of the WF relation which clearly demonstrates non-Fermi liquid behavior [20]. In shorter $1 \mathrm{D}$ constrictions there is no accepted microscopic mechanism for the 0.7 structure. One theoretical prediction [21] for the thermal conductance, that there are structures in $\kappa /\left(L_{0} T\right)$ at $G_{0} / 4$ and $3 G_{0} / 4$, has not been observed in this study. In the Landauer-Büttiker formalism the thermal conductance is calculated [19] to be $\kappa=T G_{0}\left(k_{B} / e\right)^{2} \times$ $\int_{0}^{\infty} t(E) \partial f / \partial E \epsilon^{2} d E$, where $\epsilon=\left(E-E_{F}\right) / k_{B} T, f(\epsilon)=$ $[\exp (\epsilon)+1]^{-1}$ is the Fermi function, and $E_{F}$ is the Fermi energy. In a phenomenological model [8] assuming a density dependent spin gap, calculations of the electrical conductance $G=-G_{0} \int_{0}^{\infty} t(E) \partial f / \partial E d E$ show some similarity to $G\left(V_{g}\right)$ measurements of the 0.7 structure. We find that calculations using such a model give a structure at $\kappa=L_{0} T \times 0.7 G_{0}$; that is, the Wiedemann-Franz relation is obeyed and it is not possible to obtain simultaneous plateaus at $G=0.7 G_{0}$ and $\kappa=L_{0} T\left(G_{0} / 2\right)$.

Experimentally, a plateau at $e^{2} / h=G_{0} / 2$ has been measured in the electrical conductance of low density wires [10] and longer wires [8], but it is not known whether this is a full spin polarization or some other state. A mechanism for this $e^{2} / h$ plateau in $G\left(V_{g}\right)$ has been put forward by Matveev [22], where the 1D wire is modeled as a Wigner crystal in which there are separate charge and spin degrees of freedom. The electrical resistance has contributions from both, with the spin contribution being temperature dependent and going to zero when $T \rightarrow 0$. Within this model, the thermal conductance is expected [23] to show a half plateau at $\kappa /\left(L_{0} T\right)=G_{0} / 2$.

In summary, we have demonstrated a new technique to measure the rise in temperature (typically $10-100 \mathrm{mK}$ ) of $\sim 2 \times 10^{5}$ electrons in a box, due to the heat flow in and out of the box through ballistic 1D conductors. The heat flow into the box through one of the 1D conductors is measured, and its thermal conductance characteristics $\kappa\left(V_{g}\right)$ have been deduced. For $G>G_{0}$ the WiedemannFranz relation is obeyed, in line with theoretical expectations. For $G<G_{0}$ there is no analogue in $\kappa\left(V_{g}\right)$ to the 0.7 structure in $G\left(V_{g}\right)$, but a half plateau is observed at $\kappa=$ $L_{0} T\left(G_{0} / 2\right)$ in all four samples. This half plateau, which is measured in both the linear and nonlinear regimes, should be considered in future theoretical models of the 0.7 structure.

This work was supported by the Engineering and Physical Sciences Research Council (UK).

[1] B. J. van Wees et al., Phys. Rev. Lett. 60, 848 (1988).

[2] D. A. Wharam et al., J. Phys. C 21, L209 (1988).

[3] L. W. Molenkamp et al., Phys. Rev. Lett. 68, 3765 (1992).

[4] J. B. Pendry, J. Phys. A 16, 2161 (1983).

[5] L. G. C. Rego and G. Kirczenow, Phys. Rev. Lett. 81, 232 (1998); Phys. Rev. B 59, 13080 (1999).

[6] K. Schwab et al., Nature (London) 404, 974 (2000).

[7] K. J. Thomas et al., Phys. Rev. Lett. 77, 135 (1996); Phys. Rev. B 58, 4846 (1998).

[8] D. J. Reilly et al., Phys. Rev. B 63, 121311(R) (2001).

[9] S. M. Cronenwett et al., Phys. Rev. Lett. 88, 226805 (2002).

[10] K. J. Thomas et al., Phys. Rev. B 61, R13365 (2000).

[11] S. Nuttinck et al., Jpn. J. Appl. Phys. 39, L655 (2000).

[12] P. Roche et al., Phys. Rev. Lett. 93, 116602 (2004).

[13] A. Kristensen et al., J. Appl. Phys. 83, 607 (1998).

[14] N. J. Appleyard et al., Phys. Rev. Lett. 81, 3491 (1998).

[15] N. J. Appleyard et al., Phys. Rev. B 62, R16275 (2000).

[16] N. J. Appleyard et al., Physica (Amsterdam) 6E, 534 (2000).

[17] M. Pioro-Ladriere et al., Phys. Rev. B 72, 115331 (2005).

[18] Bias cooling shifts the definition voltage from 0 to $0.3 \mathrm{~V}$.

[19] H. van Houten et al., Semicond. Sci. Technol. 7, B215 (1992).

[20] R. Fazio et al., Phys. Rev. Lett. 80, 5611 (1998).

[21] T. Rejec et al., Phys. Rev. B 65, 235301 (2002).

[22] K. A. Matveev, Phys. Rev. Lett. 92, 106801 (2004).

[23] K. A. Matveev (private communication). 